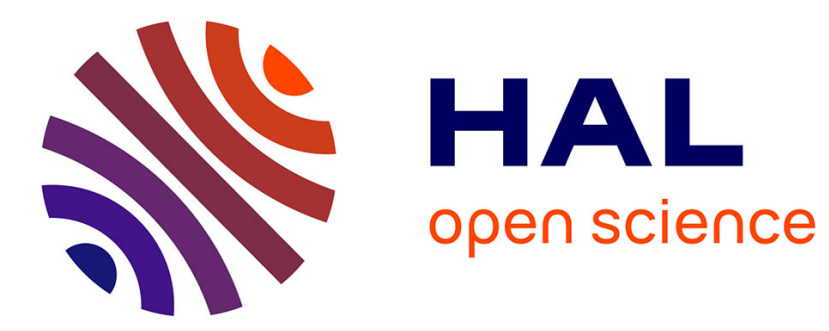

\title{
Ontologies : antinomies, contradictions et autres difficultés épistémologiques du concept \\ Jean Robillard
}

\section{To cite this version:}

Jean Robillard. Ontologies : antinomies, contradictions et autres difficultés épistémologiques du concept. STICEF (Sciences et Technologies de l'Information et de la Communication pour l'Éducation et la Formation), 2004, 11, 17 p. hal-00696355

\section{HAL Id: hal-00696355 \\ https://hal.science/hal-00696355}

Submitted on 11 May 2012

HAL is a multi-disciplinary open access archive for the deposit and dissemination of scientific research documents, whether they are published or not. The documents may come from teaching and research institutions in France or abroad, or from public or private research centers.
L'archive ouverte pluridisciplinaire $\mathbf{H A L}$, est destinée au dépôt et à la diffusion de documents scientifiques de niveau recherche, publiés ou non, émanant des établissements d'enseignement et de recherche français ou étrangers, des laboratoires publics ou privés. 


\title{
Ontologies : antinomies, contradictions et autres difficultés épistémologiques du concept
}

\author{
Jean ROBILLARD [Télé-université, Université du Québec]
}

RÉSUMÉ : Cet article a pour objet d'étude le concept d'ontologie usité en sciences cognitives (SC). La logique du concept et sa portée méthodologique sont ce qui retiendra notre attention. Notre thèse est que le concept SC d'ontologie, à part le fait qu'il heurte la sensibilité philosophique, et en plus d'être un concept dont les visées épistémologiques sont ambitieuses, est un concept autocontradictoire.

- MOTS CLÉS : Ontologie, engagement ontologique(critère quinien), sciences cognitives, ingénierie cognitive, épistémologie, catégorisation (théorie de la), théorie poppérienne des trois mondes

ABSTRACT : This article studies the concept of ontology used in cognitive science (CS). The logic of the subject and its methodological significance will be given the most attention. The thesis is that apart from having high epistemological aims and running counter to philosophic sensibility, the concept of ontology used in CS contradicts itself.

- KEYWORDS : ontology, ontological commitment (quinian criterion of), cognitive science, cognitive engeneering, epistemology, categorization (theory of), three worlds theory (popperian)

- $\quad$ 1. Introduction

- $\quad$ 2. Ontologie : de quoi parle-t-on ?

- $\quad 3$. La théorie des trois mondes de Popper

- $\quad$ 4. Ontologie poppérienne et ontologie SC

- $\quad$ 5. Conclusion

- $\quad \underline{\text { Références }}$

\section{Introduction}

Qu'est-ce qu'une ontologie ? Au-delà de la querelle classique entre les tenants du réalisme et ceux du nominalisme, en philosophie contemporaine cette question commande généralement une réponse assez nette, que signale d'ailleurs l'étymologie du mot : une ontologie est fondamentalement un discours (un logos) portant sur ce qui est ou sur ce qui existe (ontos). Remarquons immédiatement que cela qui est ou cela qui existe, est ou existe à proportion de sa disposition à être l'objet d'un discours - que l'on présumera rationnellement structuré. On voit donc que pour le philosophe l'être se dévoile par et dans le dire $^{1}$. Or, pour le chercheur en sciences cognitives - au sens générique du terme - la réponse est fort différente. Il existe bien sûr des définitions assez bien acceptées du concept d'ontologie et nous en examinerons une en raison de son exemplarité. Mais les liens qu'elles entretiennent avec le concept philosophique sont plutôt lâches et doivent être réévalués.

L'objet de cet article consiste en une analyse du concept d'ontologie tel qu'il apparait en sciences cognitives. Il faut toutefois noter que mon ambition n'est pas de proposer une analyse détaillée de tous les usages dont il est fait en ce domaine, cela dépassant mes capacités et compétences. Je ferai dans un premier temps le rappel d'une thèse, celle de J. Sowa, et la critiquerai du point de vue de sa cohérence et de sa portée épistémologique. Dans un deuxième temps, je résumerai les thèses ontologiques de Karl 
Popper et ferai de même avec certaines critiques qui lui ont été adressées. Nous verrons que ces thèses, réunies par Popper en une théorie dite des trois mondes, et dont le destin a voulu qu'elles soient rejetées puis quasiment oubliées, ont plusieurs points en commun avec celles qui prennent la défense de ce que l'on appelle "ontologie" dans les sciences cognitives. À cet égard, je soutiendrai que les critiques qui furent adressées à la théorie de Popper peuvent être transposées, pour l'essentiel, à une critique de ce dernier concept d'ontologie. Ce recours à une analyse comparative me permettra, en dernier lieu, de mettre en relief les principales difficultés de cette théorie de l'ontologie des sciences cognitives, du double point de vue épistémologique et méthodologique, en indiquant en quoi les erreurs inscrites dans le cœur même de la logique de ce même concept d'ontologie, sont de nature à discréditer les objectifs propres qui lui sont assignés. La thèse que je veux ici soutenir est que ce concept d'ontologie est autocontradictoire. Ce qui entraîne que toute théorie misant sur ce dernier est au mieux indécidable, au pire, fausse.

\section{Ontologie : de quoi parle-t-on?}

Il arrive souvent que les auteurs en sciences cognitives (SC) rappellent succinctement une définition possible, du point de vue philosophique, du concept d'ontologie, puis s'en servent comme d'un paradigme pour asseoir la définition du concept usité en SC. La définition que j'en ai moi-même donnée en ouvrant ce texte en est un exemple - un peu atypique en SC, il est toutefois vrai - . Mais les rappels qu'ils font de l'histoire de la pensée philosophique pèchent généralement par quelques lacunes importantes. En particulier, celle-ci que pour les philosophes, de quelque obédience qu'ils soient, toute ontologie n'a de sens qu'en rapport avec une théorie de la connaissance, c'est-à-dire, comme déjà le savait Aristote, un rapport établissant la différence entre la nature physique des objets sur lesquels s'exerce la science et la connaissance, et leur être métaphysique - distinguo qui a depuis été souvent réaffirmé, au demeurant, afin de soustraire la métaphysique de la réflexion philosophique valable - . Car si connaître consiste en une activité permettant la production de nouvelles connaissances ou encore l'amélioration (par exemple en augmentant la précision des concepts) de la représentation que l'on se fait de la réalité (ses parties observables comme non directement observables, etc.), alors les objets de la connaissance sont des construits qui représentent le monde tel qu'il est possible de le connaitre ; "représentation" étant ici un concept sémantiquement lié à la modalité du possible ${ }^{2}$. Entre les deux existe donc une relation telle qu'à tout élément $x$ de la classe $\mathrm{X}$ des objets du réel correspond au moins un élément $y$ de la classe $\mathrm{Y}$ de toutes les représentations possibles de $\mathrm{X}: \forall x \subset \mathrm{X}, \exists y \subset \mathrm{Y}$ t.q. $\mathrm{F}(x)=y$ donné par $\langle x, y>\subset \mathrm{F}(x)$. Notons que ces classes ne sont pas limitées ni en nombre, mais elles sont dénombrables ( si $x \subset \mathrm{X}$, alors $x+1 \subset \mathrm{X}$ ), ni en termes de nombre d'éléments qu'elles contiennent $\left(\mathrm{X}=\left\{x_{1}, x_{2}, \ldots, x_{n}\right\}\right.$, $\left.\mathrm{Y}=\left\{y_{1}, y_{2}, \ldots, y_{n}\right\}\right)$, ni ne sont restreintes quant à leur développement dans le temps, car elles peuvent être transformées grâce aux avancées de la connaissance scientifique, par exemple. Le rapport entre théorie de la connaissance (appelée classiquement épistémologie) et théorie du monde (ou ontologie) est alors évident : il serait en effet absurde de parler de la connaissance ne serait-ce que comme possibilité $d u$ connaître si cela ne signifiait pas parler de la connaissance de ce qui peut être connu (et peu importe comment $^{\underline{3}}$ ). Pour les philosophes des sciences, en majorité, une ontologie est ainsi une dimension implicite, ou rarement explicitée, de l'épistémologie. Mieux : à toute science correspond une ontologie qui lui est propre et qui la distingue des autres. Quand, par exemple, une science caractérise l'objet de son investigation en définissant une certain nombre de propriétés (empiriques, logiques, etc.), elle construit certes de cet objet une représentation, mais elle désigne aussi et littéralement les conditions de l'existence de cet objet du point de vue jugé le plus utile à la poursuite de son objectif particulier à ce moment-là. Pour tout $x, \mathrm{~F}(x)$ est vrai si et seulement si sont satisfaits les critères permettant l'identification, dans l'objet, des propriétés retenues aux fins de l'analyse au temps $t$. Voilà d'ailleurs, et vite fait pour l'instant, une interprétation de la thèse de Quine [Quine53] sur le critère d'engagement ontologique des théories, 
thèse selon laquelle "être, c'est être une variable liée" ${ }^{4}$, ce qui peut être interprété en disant que l'existence, en tant que propriété méta-physique, est une construction théorique nécessaire à la science, du point de vue de la pragmatique (i.e. les valeurs attribuées aux probabilités d'application des théories) autant que de celui de la logique et de la sémantique des théories ${ }^{5}$.

Il n'est par contre pas certain que les chercheurs en SC aient du concept d'ontologie une représentation identique, ni même compatible, et ce, même si le précédent paragraphe faisait appel à ces notions couramment utilisées en "analyse ontologique" comme, par exemple, la dimension de la représentationnalité des connaissances, cruciale en SC, ou la formalisation (au moyen surtout de la logique du premier ordre). Or, il faut rappeler que les chercheurs en SC sont intéressés par le concept dans la mesure où leur but est exclusivement le développement de systèmes informatiques de gestion des connaissances, ou de concevoir des systèmes à base de connaissances de plus en plus puissants. En bref, ce qui est visé, c'est une ingénierie des connaissances. Et un tel projet repose fondamentalement sur le niveau d'abstraction et de formalisation qu'il serait souhaitable de fixer à l'analyse des connaissances et il n'est pas sûr que les niveaux les plus élevés (top-level ontology, par exemple ; v. [Floridi99]) soient les plus prometteurs. Quoi qu'il en soit, ce qui est d'un intérêt philosophique et épistémologique plus certain, c'est comment sont articulées et structurées les théories de la connaissance adoptées et mises en œuvre dans ce domaine de la recherche.

Prenons à titre d'exemple, parmi de nombreux autres possibles, la proposition avancée par J. Sowa ${ }^{6}$ (http ://www.jfsowa.com/ontology/index.htm). Son intérêt réside dans l'effort de synthèse dont elle fait preuve.

The subject of ontology is the study of the categories of things that exist or may exist in some domain. The product of such a study, called an ontology, is a catalog of the types of things that are assumed to exist in a domain of interest $\boldsymbol{D}$ from the perspective of a person who uses a language L for the purpose of talking about $D$. The types in the ontology represent the predicates, word senses, or concept and relation types of the language $\boldsymbol{L}$ when used to discuss topics in the domain $\boldsymbol{D}$. An uninterpreted logic, such as predicate calculus, conceptual graphs, or KIF, is ontologically neutral. It imposes no constraints on the subject matter or the way the subject may be characterized. By itself, logic says nothing about anything, but the combination of logic with an ontology provides a language that can express relationships about the entities in the domain of interest. An informal ontology may be specified by a catalog of types that are either undefined or defined only by statements in a natural language. A formal ontology is specified by a collection of names for concept and relation types organized in a partial ordering by the type-subtype relation. [...].

Voyons que pour Sowa une ontologie est ni plus ni moins qu'une taxinomie ${ }^{7}$, qu'une classification de "choses" d'un domaine de connaissances quelconque $\boldsymbol{D}$ (empirique ou formel), dont la critériologie implicite est en réalité une pragmatique linguistique fondée sur l'unique règle visant à garantir l'adéquation de ces choses au langage $\boldsymbol{L}$ utilisé afin de fournir de ce domaine une représentation conceptuelle effectuée sur la base : 1) du sémantisme des termes ${ }^{\underline{8}}$ - bien que ce ne soit pas clair de prime abord - servant à nommer des classes catégorielles disjointes ; 2) du système de relations formelles que ces classes entretiennent entre elles; et 3) de l'usage, par un locuteur qu'on imagine être compétent, de ces termes dans un effort de description du domaine ${ }^{9}$. Or, ce qui est frappant, ici, également, c'est que ce que j'appellerais le postulat de représentationnalité des termes catégoriels qui les place, ces termes, dans un rapport ontique et non pas ontologique aux objets réunis en classes disjointes, dans la mesure où, d'une part, ils sont littéralement pris en charge par le système formel dont ils héritent la syntaxe, et que, d'autre part, ils ont pour fonction de signifier le contenu épistémique des classes catégorielles composé des prédicats, du sens des mots, des types de concepts et des relations repérables au sein du langage $L^{\underline{10}}$. S'inscrit alors, au sein même du système de relations formelles, une relation langage/métalangage déterminant ainsi le caractère analytique du métalangage descriptif. L'exigence de 
la correspondance des choses aux termes décrivant leurs classes d'appartenance est en ce sens davantage qu'une platitude : c'est une impossibilité logique dans la mesure où cette partie de la thèse ne vise qu'à établir la référentialité des termes descriptifs, c'est-à-dire à leur assurer un lien de correspondance aux choses auxquelles ils se rapportent par le biais d'un langage servant à discuter des thèmes du domaine $\boldsymbol{D}$ : or ces termes servent à nommer des classes de choses, à la manière, oserait-on dire, des noms propres, ils se situent, sur le plan sémantique, à un niveau qui n'est déjà plus celui de l'observation des choses. Il ne sert de rien, dans un tel contexte, d'insister sur le fait que les termes catégoriels représentent ou identifient des "choses" qui existent ou dont on pourrait dire qu'elles existent, puisque ce n'est pas de l'existence de celles-ci dont il s'agit mais de la justesse de la description qu'en offrent leurs noms. De cela découle que la véritable théorie qui est ici proposée est une théorie de la description, bien qu'elle soit éminemment incomplète, pas une ontologie (sur les problèmes logiques et sémantiques de la description, voir entre autres : [Carnap56], [Quine60], [Davidson84] et [Neale93]). Mais faute d'en avoir réalisé pleinement la nature, Sowa, comme d'autres, reproduit des erreurs conceptuelles que, pourtant, les philosophes ont depuis longtemps corrigées.

D'autre part, la relation que Sowa établit entre le domaine $\boldsymbol{D}$, le langage $\boldsymbol{L}$ et la logique, n'est ni claire, ni, surtout, assurée. En effet, dans un premier temps, la thèse selon laquelle la logique serait ontologiquement neutre est une thèse difficile à soutenir sans risquer de subir un croc-en-jambe de la part de l'épistémologue en service. Car en tant que le critère quinien d'engagement ontologique est vrai, et Church [Church58] en a en outre démontré la portée générale d'un point de vue logique, c'est que, comme ce dernier le rappelait, une variable liée ne peut être vide et cela est tel en fonction des règles de quantification (universelle ou existentielle, il n'importe). Or, la logique utilise abondamment des variables et les quantificateurs. Il faut alors conclure que le théoricien qui se sert de la logique se doit d'en respecter les engagements ontologiques. Quels peuvent-ils être?

Contrairement à la thèse naïve critiquée ici, ils ne concernent pas le monde objectif ou empirique (à moins d'une décision prise à cet effet par le théoricien et établissant a priori une convention ${ }^{11}$ ), mais plutôt le "monde" des vérités logiques et autres "êtres" abstraits et règles de toutes sortes qui peuplent la théorie de la logique. Écrire par exemple $: \forall p, \forall q,((p \rightarrow q) \& \sim p) \rightarrow \sim q$, c'est nécessairement admettre que les variables propositionnelles $\mathrm{p}$ et $\mathrm{q}$ sont applicables à toute proposition de quelque langage que ce soit, formel ou naturel, et que la règle du raisonnement par modus tollens sera toujours vraie ; c'est, de plus, et sans pour autant devoir faire un acte de foi réaliste au sens médiéval du terme, respecter l'engagement suivant lequel il existe des propositions au sens de la logique. Or, si la logique n'est pas ontologiquement neutre - comment, d'ailleurs, pourrait-on définir un concept de neutralité ontologique d'une théorie depuis Quine? - , elle ne peut jouer le rôle de simple matrice formelle à la représentation des connaissances, tel que le propose aussi Sowa. Car c'est sur cet argument du caractère de neutralité ontologique de la logique que repose ses thèses de la représentativité des ontologies et du sémantisme catégoriel développées grâce à une syntaxe "pure" que fournirait la logique.

Une autre dimension importante de la théorie de Sowa, et de plusieurs autres, est son platonisme inavoué. Rappelons que Platon croyait en l'existence d'un monde peuplé de formes idéelles, les seules dont on pouvait dire qu'elles existaient réellement; elles étaient les seules à partir desquelles l'on pouvait donc vérifier la vérité de nos connaissances. Les autres formes, particulièrement celles de la nature, en étaient pour lui que de simples copies, d'où leur statut inférieur par rapport aux idées qu'elles reproduisaient sans précision. Cette thèse avait vocation à résoudre un problème épistémologique important, celui de concilier le fait que les choses qui se ressemblent n'ont pas toutes et exactement les mêmes caractéristiques observables et pourtant peuvent être reconnues comme participant de la même "espèce" : tous les sapins se ressemblent, mais ils ne sont pas tous parfaitement identiques. Les œuvres d'art, y compris la poésie, quant à elles n'étaient pas en reste, elles qui n'étaient que pâles copies de copies. L'ordre logique de l'existence posait donc le principe de la primauté des idées sur les autres niveaux de 
l'existence. Plus on s'éloignait du monde des idées, plus on s'éloignait de la vérité ; plus la connaissance perdait de sa précision et de son acuité. (Il vaut maintenant la peine de rappeler que, pour la philosophie médiévale, le réalisme des idées est platonicien et que le nominalisme ne l'est pas, celui-ci refusant d'attribuer une quelconque existence autonome aux idées ou aux concepts en tant qu'êtres abstraits).

Or, quand je dis que la thèse de Sowa est platonicienne, je ne prétends pas que l'auteur lui-même défend jusqu'au bout l'ontologie platonicienne. Ce que je peux dire, par contre, c'est que l'idée de Sowa selon laquelle une ontologie SC peut représenter toutes les choses d'un domaine $\boldsymbol{D}$ descriptible dans un langage $\boldsymbol{L}$, est une idée qui exprime très précisément une conception idéelle de l'organisation des connaissances : en effet, penser qu'un langage quelconque puisse décrire toute réalité quelle qu'elle soit est non seulement attribuer à ce langage une puissance inconnue à ce jour, mais c'est surtout présupposer que pour ce faire il suffira de classer les choses adéquatement (i.e. conformément aux ressources logiques et sémantiques du langage, ce que l'on sait être insuffisant, voire incorrect, depuis A. Tarski [Tarski72]) et de nommer ces classes de choses de telle manière que ces noms exprimeront des concepts suffisamment généraux pour pouvoir signifier les choses ainsi classées malgré leur individualité et leurs différences. La typologie linguistique, définie par Sowa comme étant donnée par les "predicates, word senses, or concept and relation types of the language $L^{\prime \prime}$ n'est donc en ce sens qu'une autre façon de dire que les ressources logiques et sémantiques d'un langage quelconque suffisent mimétiquement à la tâche de la représentation formelle du monde - d'où on peut par ailleurs conclure que le recours à la logique comme de n'importe quel autre technique de formalisation n'est pas supplémentairement très utile. De plus, cette typologie est incertaine dans la mesure où lui fait défaut une règle interprétative permettant à l'usager du langage visé par la thèse de déterminer la portée sémantique des contenus intensionnels des classes - que cette typologie mentionne explicitement. Et parce que ce qui compte, en bout de piste, c'est cette représentation à base de description, on est devant une théorie de la connaissance qui prône la primauté de la représentation idéelle des objets de connaissance et le mimétisme comme méthode d'accès aux réalités non idéelles. Or, comme l'objectif manifeste de Sowa est de proposer une méthode de classification, cela en fait un platonisme méthodologique. Ce qui est son droit le plus strict. On retrouve cette même approche chez Floridi ([Floridi99], pp. 112-115), un philosophe.

Mais le malheur avec cette dernière, c'est qu'elle repose sur une double difficulté majeure :

- à partir de quoi décide-t-on d'inclure ou d'exclure une "chose" d'une classe, c'est-à-dire quels critères utiliser afin de réunir en une même classe des objets quelconques ? Et subsidiairement, que classe-t-on, réellement : des objets ou des descriptions d'objets énoncées dans quel type de langage et iveau ?

- et à partir d'une collection de combien d'exemplaires de la "même" chose peut-on parler de l'existence d'une telle classe $\mathrm{e}^{12}$ ? Et ceci n'a pas pour but de ramener sur le devant de la scène la querelle millénaire entre le réalisme et le nominalisme. Il s'agit plutôt d'un problème épistémologique et méthodologique d'une très grande importance mais que, malheureusement, je devrai laisser en suspens, faute d'espace pour en traiter.

\section{La théorie des trois mondes de Popper}

Je vais maintenant procéder à une synthèse de la théorie des trois mondes de Popper en en faisant ressortir les principales caractéristiques. Cela me permettra ensuite d'en comparer les principaux arguments aux arguments de ses détracteurs et d'expliquer pourquoi ceux-ci ont eu raison. Car, en effet, à la suite de ces critiques, la théorie des trois mondes de Popper est presque tombée dans l'oubli, ou du moins n'est-elle guère plus qu'un objet académique qui intéressera surtout l'étudiant attiré par la totalité de la philosophie poppérienne des sciences. Pour les fins de mon analyse, je m'appuierai uniquement sur son ouvrage $L a$ connaissance objective [Popper91].

Le but de la théorie poppérienne des trois mondes est essentiellement de démontrer l'objectivité des connaissances et d'en expliquer le progrès - ce qui a pour corollaire normatif que seule la connaissance 
objective peut être jugée comme possédant quelque valeur - : "la connaissance objective, ou connaissance au sens objectif, (...) consiste dans le contenu logique de nos théories, conjectures, hypothèses [...]" ([Popper91], p. 136). Cette théorie s'oppose à celle que Popper identifie comme étant la théorie de la connaissance subjective, c'est-à-dire celle qui repose sur les dispositions subjectives à intégrer psychologiquement et passivement des contenus à partir d'expériences sensorielles et identifiées comme des contenus de croyances. La connaissance objective suppose donc l'absence du sujet connaissant. Et cela, pour deux principales raisons. La première, c'est que la connaissance objective est par définition liée à la présence d'un langage à partir duquel peuvent être décrits les contenus logiques de la connaissance objective ; ce langage, au niveau inférieur, permet la communication et cette fonction est nécessaire mais non suffisante à l'existence ou à l'avènement de la connaissance objective. Du point de vue de la communication, en effet, le langage est accessoire à l'objectivité de la connaissance en ce sens que les mots du langage et leur signification ne sont que des conventions utiles à l'établissement de la communication, c'est-à-dire de l'intersubjectivité nécessaire à la discussion critique en tant que médium de l'objectivité de la connaissance. La seconde, c'est que ces contenus théoriques ne sont pas vrais en soi. En effet, et sans entrer dans l'analyse du problème de la vérité dans l'épistémologie poppérienne, disons que pour Popper la vérité n'est jamais atteinte (il a d'ailleurs introduit le concept de "vérisimilitude" défini à partir de la probabilité qu'un énoncé théorique a d'être plus ou moins vrai) : seulement, grâce à la critique rationnelle, une théorie ne peut que comporter moins d'erreurs logiques que celles qui l'ont précédée. Car pour Popper, la seule manière de rendre compte justement du progrès ou du développement de la connaissance scientifique (ou ordinaire), c'est de considérer que ce développement procède par élimination des erreurs contenues dans les théories ${ }^{13}$, et lorsque ces erreurs sont corrigées les théories sont alors de facto améliorées ou remplacées : seule la plus apte "survivra" au processus. Cette procédure de falsification des théories assure le progrès de la connaissance scientifique et garantit les conditions de son objectivité. Or, pour ce faire, les théories doivent être critiquées du point de vue de leurs contenus logiques - ce qui veut dire que la critique porte sur les contenus objectifs des théories puisque les contenus logiques sont reconnus pour leur objectivité, et Popper ne fait aucun mystère à cet égard de son appréciation des thèses de Frege et de Bolzano à propos de l'existence de tels contenus et de leur statut ontologique - et lorsque leurs erreurs sont débusquées grâce à la critique rationnelle, lorsque leurs thèses sont falsifiées puis remplacées par d'autres plus précises, le processus recommence. La connaissance objective a ce caractère d'être le fruit de conjectures réfutables. Mais jamais une théorie scientifique n'atteint le stade de la pleine vérité de son contenu ${ }^{14}$.

Retenons alors que la théorie poppérienne des trois mondes est liée à

- une théorie de l'objectivité de la connaissance scientifique opposée aux théories subjectivistes des connaissances comme contenu de croyances ;

- à une théorie réaliste (au sens médiéval) du langage dans l'organisation des connaissances dans la mesure où celles-ci sont ontologiquement des entités abstraites que dévoile la discussion critique ;

- et à une théorie du développement de la connaissance au moyen d'une procédure de falsification.

Or, la théorie des trois mondes est à strictement parler une ontologie de la connaissance objective. Chacun des trois mondes est pour ainsi dire peuplé d' "êtres" propres. Le premier monde, ou "monde physique", est constitué de ce que Popper appelle les "états physiques" ([Popper91], p. 247), c'est-à-dire autant des actions humaines que de tout ce qui est matériellement organisé, comme les roches et les cathédrales. Le deuxième monde, ou "monde mental", est celui qui comprend les "états mentaux", c'est-à-dire les différents états liés à la psychologie subjective. Enfin, le troisième monde est "le monde des intelligibles, ou des idées au sens objectif; c'est le monde des objets de pensée possibles : le monde des théories en elles-mêmes; des argumentations en elles-mêmes; et des situations de problèmes en elles-mêmes" (ibid.). Or, ce troisième monde contient aussi, bien que Popper se soit récusé plus tard à ce sujet, les documents écrits tels les livres, les revues, et les auvres d'art, bref toutes les productions culturelles fixées sur un support matériel quelconque. 
Ce qu'il faut maintenant remarquer c'est la fonction médiatrice du deuxième monde qui sert à lier entre eux le premier et le troisième monde. Ce rôle est extrêmement important, étant donné qu'il est directement impliqué dans l'objectivation des connaissances scientifiques.

Ce que l'on peut appeler le deuxième monde - le monde de l'esprit - s'affirme de plus en plus, [...], comme le lien entre le premier et le troisième monde : toutes nos actions dans le premier monde sont influencées par la saisie que notre deuxième monde a du troisième. C'est pourquoi il est impossible de comprendre l'esprit humain et le moi humain sans comprendre le troisième monde [...] ; c'est pourquoi il est impossible d'interpréter le troisième monde comme la simple expression du second, ou le second comme le simple reflet du troisième. (Op. cit., p. 236)

La suppression du sujet épistémique - et l'élimination de la théorie classique de la connaissance en tant que contenu d'une croyance - passe donc par la capacité de l'esprit humain à faire se correspondre les contenus objectifs de la connaissance logés dans le troisième monde et les manifestations physiques du monde physique apparaissant dans le premier. (Ce qui déjà pose un premier problème de cohérence interne à l'argument poppérien.) Et si cela est tel, c'est que le deuxième monde rend possible l'utilisation du langage dans sa forme supérieure ${ }^{15}$ et donc que les théories qui traitent des objets du premier monde peuvent être mises à l'épreuve de la critique rationnelle au moyen de la falsification. Car le langage est le "médium de l'argumentation, de la discussion critique" (op. cit., p. 221) et en ce sens, le troisième monde "est un sous-produit du langage" (ibid.). Mais voilà que le processus d'objectivation des connaissances, une activité humaine ([Popper91], p. 252), confirmera pour Popper l'autonomie du troisième monde par rapport aux deux autres; et, du coup, cela confirmera la thèse selon laquelle les idées, théories et argumentations qui peuplent le troisième monde possèdent une vie autonome et indépendante des deux autres. L'argument servi par Popper à cet effet est celui du sens de la notion de découverte en tant que découvrir une nouvelle connaissance objective cela présuppose que celle-ci préexistait à l'activité humaine ayant mené à sa découverte. Contrairement à Platon, l'ordre logique de l'existence proposé par Popper ne laisse aucunement entendre que les idées préexistent à la réalité physique et matérielle des objets du premier monde, mais qu'elles préexistent à la saisie humaine de leur existence au moyen de la découverte effectuée grâce à une méthode d'élimination des erreurs contenues dans les théories. L'autonomie des contenus objectifs de la connaissance se mesure essentiellement à leur potentiel d'être découverts puis critiqués.

Les critiques adressées à Popper ont été nombreuses et sans merci (v. entre autres [Carr77], [Church84], [Cohen80], [Gilroy85], [Keuth74], [Klemke79], [Krausz74]). Elles ont, selon divers points de vue, débattu des antinomies de sa théorie des trois mondes et des contradictions auxquelles elle menait.

L. J. Cohen [Cohen80] l'a attaquée sur plusieurs fronts. Son article présente une série de problèmes qui lui sont immanents, qu'il gradue selon leur niveau croissant de difficulté. L'un des plus difficiles concerne la thèse avancée par Popper selon laquelle le troisième monde contient toutes les conséquences de n'importe laquelle théorie scientifique (puisque toutes ces conséquences préexistent à la découverte qu'en font les êtres humains au moyen de la critique) et que leur découverte équivaut à l'augmentation du volume de connaissances désormais disponibles. Cette thèse est certainement le socle sur lequel Popper fait en grande partie reposer l'autonomie du troisième monde. Or, nous dit Cohen, le fait que ces conséquences n'aient pas été toutes découvertes ne veut rien dire d'autre que ce progrès des connaissances n'est pas un processus propre au troisième monde, mais qu'il s'agit bien davantage d'une question liée à la subjectivité des chercheurs dans la mesure où il n'est pas possible de dire autre chose que si les connaissances qui restent à découvrir ne sont pas encore découvertes, c'est uniquement parce que l'activité cognitive des chercheurs n'y est pas encore parvenu, ou, en d'autres termes, que les problèmes irrésolus le sont uniquement dans l'esprit ("mind") des chercheurs qu'ils se trouvent être traités ([Krausz74] se sert du même argument). La critique de Cohen porte donc ici sur le caractère immatériel des entités composant le troisième monde de Popper et sur le fait qu'il est logiquement impossible de déduire de l'absence de nouvelles conclusions qu'il y en aura, sinon qu'en faisant un raisonnement ex 
ante ou antérograde a posteriori; sa critique porte non sur leur caractère d'être des entités abstraites. C'est le réalisme (au sens médiéval) de Popper qui est remis en cause par cet argument. La thèse affirmant l'autonomie du troisième monde est ainsi remise en question, son ontologie l'est par conséquent.

Cet argument est renforcé sur le plan de sa logique lorsque, avec Cohen (ibid.), l'on considère une situation où, à propos d'un domaine de recherche quelconque, une théorie $T$ est, en vertu des critères poppériens, la meilleure à ce jour. Supposons de plus qu'un seul chercheur se soit penché sur la théorie $T$ et que $s a$ théorie $\left(T^{\prime}\right)$ dit que $T$ n'est pas la meilleure théorie à ce jour. Alors,

It seems undeniable then that T' deserves a place in the third world, alongside $T$, and indeed that $T$ ' is itself the best theory so far proposed. No doubt $T$ ' is false, but $T$ may well be false also, so there is nothing to choose between $T$ and $T$ ' on this score. Accordingly it looks as though the third world declarations of theory merit are potentially incoherent. ([Cohen80], p. 179)

Cette incohérence pourrait être renversée, poursuit Cohen, par l'ajout d'un postulat particulier à la thèse poppérienne, stipulant que les théories de second niveau comme $T$ ' doivent être bannies du troisième monde en vertu de considérations liées par exemple à l'autonomie des théories de premier niveau. Mais comment alors concilier le fait que ces théories de second niveau contribuent également au progrès des connaissances, même si elles sont fausses, et le fait que la thèse de Popper dans son pluralisme même ne pose aucune interdiction de ce genre ? Sans doute en postulant l'existence d'un quatrième monde qui accueillerait les théories de second niveau. Mais alors s'ensuit nécessairement une régression ad infinitum dans la mesure où pour chacun des niveaux, un niveau d'ordre immédiatement supérieur devra être postulé afin de toujours éliminer l'incohérence en question.

It follows that the third world can hardly be supposed uniformly capable of including, whenever a new theory is fed into it, the fact that it is consistent or inconsistent, as the case may be; or we should be attributing to the third world a capacity that is demonstrably impossible. ([Cohen80], p. 178)

Ces quelques critiques atteignent le cœur même de la théorie des trois mondes de Popper et la réduisent à l'état de curiosité philosophique. Popper souhaitait fournir une ontologie des connaissances à ce point forte qu'elle eût permis d'embrasser éventuellement l'ensemble de la culture. Mais il a échoué. Et ce n'est pas faute de n'avoir pas essayé.

\section{Ontologie poppérienne et ontologie SC}

En quoi l'ontologie poppérienne se compare-t-elle à l'ontologie SC et que peut-on conclure de cette comparaison ? Si la première est liée à la science (ou la connaissance scientifique) et lui est destinée ; et si la seconde est liée à la technologie de l'information et lui est également destinée, alors cette différence les rend-elles incomparables?

Je reviendrai à la première question dans un moment car la deuxième requiert une réponse immédiate. Pour ce faire, il faut dans un premier temps mesurer la thèse suivante, émise par Mario Bunge : "La technologie hérite l'ontologie de la science, et produit à son tour sa propre ontologie." ([Bunge83], p. 227) Rapporté à un cadre quinien, cela veut dire, plus explicitement, ceci : la technologie partage avec la science les engagements ontologiques de cette dernière et en produit d'autres qui lui sont propres du fait de son domaine particulier d'action. Or, il faut bien comprendre ici que le concept d'engagement ontologique est entendu strictement au sens de Quine [Quine53] et non à celui des SC où on en fait quelque chose comme une norme d'autosatisfaction du critère de la correspondance des classes catégorielles aux "choses" qu'elles sont censées contenir. Le critère quinien d'engagement ontologique est d'abord et avant tout un critère logique, c'est-à-dire un critère s'adressant à la forme des énoncés. De 
plus, ce critère ne s'applique pas à des noms de choses, fussent-elles des catégories, mais à des variables liées (ou quantifiées) : une variable quantifiée n'est pas substituable, tandis qu'un nom l'est et cette différence rend immédiatement caduque tout prétention d'appliquer le critère quinien à autre chose qu'à des énoncés logiques quantifiés. Par exemple (repris de [Keuth74]), lorsque nous utilisons un nom comme " $a$ " il va de soi que nous reconnaissons implicitement que ce nom réfère à quelque chose. Mais ce nom appartient à un langage et si ce dernier admet l'usage de variables individuelles et de quantificateurs, alors il nous est très certainement permis d'inférer ceci : $\mathrm{F} a: a$ est $\mathrm{F} \rightarrow \exists x: \mathrm{F} x$. Si, toutefois, " $a$ " signifie le nom "Pégase" et que F est une propriété que nous pensons être possédée uniquement par Pégase et par rien d'autre, alors nous pouvons bien admettre " $\mathrm{F} a$ " comme vrai, mais " $\exists x: F x "$ sera faux car Pégase n'existe pas. C'est pourquoi le critère d'engagement ontologique s'applique aux variables liées et pas aux noms de choses $\frac{16}{\text {. }}$.

Dans cette perspective, la thèse de Sowa sur les noms de catégories de l'ontologie SC, selon laquelle ces noms jouent le rôle d'identificateurs de prédicats et d'autres propriétés logiques permises par le langage $\boldsymbol{L}$, est une thèse fausse. En tant que ces noms ne sont pas substituables, ils partagent certainement cette caractéristique des variables liées. Mais un nom, surtout utilisé comme ici comme un nom propre (ou une description définie), n'est pas identique à une variable : c'est a contrario une constante qui, sur le plan logique, n'identifie qu'une et une seule classe d'"objets ${ }^{\underline{17}}$ ", classe qui, au surplus, n'est pas définie extensionnellement mais intensionnellement. Tout ce que l'on est en droit d'attendre de cette situation, c'est à une identité logique entre le nom ' $X$ ' et la classe $X$. L'identité logique attribuée à la classe est garantie par son nom, pas par ceux de ses éléments, qui d'ailleurs en sont absents puisque sa définition est intensionnelle. Comme il advient chez Sowa, en ce qui a trait aux ontologies SC ces éléments sont généralement des propriétés sémantiques de concepts. Et en tout état de cause, une définition intensionnelle d'une classe d'appartenance utilisera les quantificateurs logiques $(\exists, \forall)$ et des variables d'individus leur seront liées afin d'identifier certaines propriétés individuelles des concepts visés. Le critère quinien d'engagement ontologique des théories pourra alors certes s'appliquer, mais il engagera alors l'ontologie SC à contracter un engagement sur l'existence du sens des concepts qu'elle lie - ce qu'identifie en réalité le nom de la classe qu'il a construite - . Et cela est d'une extrême banalité.

Donc, si les ontologies SC ont vocation, comme cela est le cas ici, à offrir une taxinomie de concepts usités dans un domaine quelconque, elles ne peuvent pas en même temps prétendre représenter l'ontologie, au sens philosophique du terme, des théories que ces concepts contribuent à structurer. Le critère quinien d'engagement ontologique ne connaît pas d'application de ce type. Or, on peut légitimement supposer qu'une taxinomie conceptuelle d'un domaine scientifique ou théorique quelconque hérite de l'ontologie de ce domaine : alors le critère d'engagement ontologique s'applique à la taxinomie en tant que technique de formalisation des relations sémantiques ou épistémiques qu'ont les concepts entre eux dans un domaine particulier. Mais là doit s'arrêter la transitivité attribuée, peut-être abusivement, au critère quinien. Car une fois un système à base de connaissances développé et mis en opération, pourra-t-on dire que ce système est soumis au critère d'engagement ontologique ? Pour répondre à cette question, il faut distinguer entre la théorie du système, de laquelle participe partiellement l'ontologie SC en tant que technique, et le système lui-même en tant que mécanisme physique résultant d'une programmation. Et comme le critère quinien est destiné aux théories, pas aux choses même intangibles comme un système d'information. Alors, rien ne distingue, sur le plan technique, une ontologie SC d'un dictionnaire ou mieux d'un thésaurus. Ils se distinguent certes par la configuration et les spécifications techniques des supports qu'ils utilisent. Mais en appliquant la méthode de l'hypertexte, un thésaurus peut très bien être "traduit" en langage informatique et se retrouver sur un cdrom. Il n'y a aucun obstacle technologique à un tel projet (qui n'est pas nouveau) $\frac{18}{\text {. }}$.

En quoi l'ontologie poppérienne se compare-t-elle à l'ontologie SC ? Il peut sembler incongru de faire une telle comparaison. Mais cette approche se justifie. Dans un premier temps, on voit assez aisément que 
les deux types d'ontologie présentent des caractéristiques propres au réalisme (au sens médiéval du terme) et au platonisme. De cela découle nécessairement que les modes de représentation et les techniques de formalisation des relations entre entités détermineront l'ordre des réalités concrètes qu'elles sont censées exprimer. Mais est-ce bien le cas ? Cela est rien moins certain, chez Sowa ou chez Popper. Mais on peut examiner la chose d'un autre angle, celui de la méthode générale.

L'on pourrait dire, en premier lieu, que ce platonisme est restreint, quant à son application, à la méthode de formalisation des bases de données sur lesquelles reposent les systèmes à base de connaissances. Ce que l'on appelle "ontologie", et L. Floridi promeut cette idée ([Floridi99], p. 110), présenterait donc des modèles de ces bases de données qui seraient alors davantage que des sources de données, d'information et de connaissances sans originalité et déterminées par les procédures d'accès qui mènent à elles, mais

"... [they] are also strategic resources whose overall purpose is to generate new information out of old data, that is, mines of that digital gold which is at the centre of most human activities. From this perspective, the infosphere is the authentic reality that underlies the physical world, and as such it has a normative or a constructionist function : a clear design of the structure of a particular domain in terms of data model or information system is either a conditio sine qua non for any implementation or, when the implementation is already available, an essential element that contributes to the proper modification and improvement of the conceptualized reality in question. Thus, the infosphere comes first in the logical order of realities, and it is the necessary conceptual environment that provides the foundation for any meaningful understanding of the surrounding world, one of its many possible implementations.

Cette thèse est intéressante à plus d'un égard. Retenons toutefois cette idée que de vieilles données inscrites dans une base quelconque, il puisse en ressortir de nouvelles informations $\underline{19}$. Or, ce que cela veut dire c'est que ces nouvelles informations étaient là avant l'intervention de l'humain qui aura questionné la base de données de manière à en faire ressortir ces nouvelles informations. Qu'elles soient le résultat d'une recherche organisée dans l'esprit de l'usager et que les algorithmes de recherche soient pour ainsi dire transparents à son usage, ne change évidemment rien : cette thèse reproduit sans peut-être même le savoir l'organisation fonctionnelle des trois mondes poppériens. La théorie qui s'en dégage à l'analyse, et qui est implicite, est que l' "infosphère" jouit de la même autonomie que celle que Popper assignait à son troisième monde; théorie qui fut battue en brèche. De plus, la médiation de l'usager est également typique du deuxième monde poppérien en ce sens qu'elle établit une relation fonctionnelle entre le système en tant que mécanisme physique et les fonctions de recherche et de génération d'informations explicitables sur le plan formel. Le sujet épistémique est évacué du processus. La psychologie individuelle n'intervient pas, n'étant pas logiquement nécessaire. Pourquoi en est-il ainsi ? Fondamentalement, parce que le platonisme, fût-il méthodologique, son idéalisme inhérent mis à part, est une théorie mimétique de la connaissance: les formes logiques des connaissances sont ce qui est reproductible tant au sens d'être reproduites dans l'esprit humain au moment de leur saisie ou de leur découverte, qu'au sens de modèles permettant l'avènement d'autres formes de connaissances dont elles sont issues.

La force particulière d'une telle théorie réside cependant dans son caractère de quasi évidence. En effet, il me semble évident que l'usager "moyen" des bases de données ou que l'habitué "moyen" de la recherche sur Internet pourraient répondre favorablement à une question leur demandant s'ils croient que l'information organisée et contenue dans les systèmes constitue un monde en soi indépendant de leur présence en tant que sujet épistémique. Cela, simplement parce que ces systèmes sont référentiellement opaques, au sens quasi logique où tout énoncé informationnel bien formé (grâce à la logique booléenne, par exemple) n'est pas substituable (toute nouvelle formulation dans une recherche produit des résultats différents, quantitativement et qualitativement, à chaque fois même s'ils sont parfois comparables), et dans la mesure où les logiciels sont en soi des outils sur lesquels très peu d'usagers peuvent intervenir en vue de les modifier, les transformer, etc. ( $c f$. les logiciels libres). Étant référentiellement opaques, ces systèmes semblent posséder une autonomie réelle, du moins par rapport aux possibles et aux probables 
actions de l'usager. Mais celles-ci sont ni plus ni moins que toujours déjà là, parce que matériellement inscrites dans le code même avec lequel les données et les informations sont structurées. Il ne faut donc pas confondre le déterminisme physique inhérent aux systèmes avec une pseudo loi déterministe de l'organisation des connaissances. Une telle loi n'existe pas. L'ontologie de Popper entendait très certainement parvenir à établir une telle loi, mais les limites épistémologiques de sa théorie en auront, une fois mises à jour et critiquées, entravé pour de bon la formulation.

\section{Conclusion}

La thèse de Sowa a le mérite de poser le problème de la définition des ontologies SC du point de vue théorique, et elle est certainement plus développée que celle de Gruber [Gruber95], selon laquelle une ontologie serait une spécification de l'ordre de la conceptualisation mise en œuvre. Dans les deux cas, il s'agit fondamentalement d'une entreprise pragmatique [Smith04], c'est-à-dire d'un cadre théorique d'actions visant l'établissement de relations plus ou moins logiquement analysables entre différents domaines d'applications. Or, de la thèse du premier on a vu que les fondements logiques et épistémologiques étaient bancals et que, en dépit de ses vœux, elle présentait davantage le visage d'une théorie incomplète de la description que d'une théorie permettant l'analyse des concepts usités dans un domaine quelconque de connaissances.

La comparaison avec la théorie des trois mondes de Popper avait pour but de dégager le portrait d'une ontologie conçue à partir d'un très petit nombre de catégories, soit trois, et que, malgré cela, les problèmes de cohérence logique et épistémologique qu'elle présente ont motivé son rejet et son abandon. Non seulement cette théorie ne pouvait fournir d'explication valable du développement des connaissances, mais elle ne pouvait certainement pas être appliquée à l'analyse des connaissances, sur le plan de leur validité interne autant que sur celui de leurs multiples relations.

Or, si d'une ontologie minimaliste sur le plan taxinomique et catégorique, en l'occurrence celle de Popper, l'on peut déduire de multiples contradictions et autres difficultés internes, il est fort possible qu'une ontologie fondée sur une taxinomie plus généreuse verra ces difficultés décuplées. L'exemple de Sowa démontre qu'il peut en être effectivement ainsi. Le problème fondamental, alors, ne réside pas tant au niveau des objectifs - analyser des concepts et leurs applications à quelque domaine que ce soit et inscrire la formalisation ainsi obtenue comme structure de représentation de "connaissances" dans un système à base de connaissances - , que dans le postulat méthodologique premier selon lequel le sens des concepts peut être catégorisé et inclus dans une classe d'appartenance. Or, on a vu qu'il fallait dans un premier temps faire le ménage, que ce postulat n'avait pas la force qu'il prétend avoir. Il fallait de plus séparer ce qui est de l'ordre de la description des concepts et ce qui est de l'ordre des actions ou des états de choses que l'on cherche à représenter; et qu'il fallait également distinguer entre différents niveaux de langage utilisés pour ce faire afin d'assurer à l'ontologie une complétude sémantique [Granger92] que la méthode actuellement ne permet pas d'atteindre. Le défaut théorique est ici celui de l'absence d'une théorie de la catégorisation et de la sémantique catégorielle qui doit obligatoirement l'accompagner.

Enfin, ce postulat méthodologique est imbriqué dans une philosophie platonicienne de la connaissance et on aura compris que le mimétisme de celle-ci posait une difficulté supplémentaire, celle de l'intelligibilité de l'ontologie : le mimétisme en question est ce qui peut être pointé comme l'élément principal dont sont issues les contradictions internes aux ontologies étudiées ici.

${ }^{[1]}$ C'est dans cette voie tracée par les philosophes depuis l'Antiquité qu'il faut dans doute interpréter le fameux aphorisme 7.0 sur et avec lequel se termine le Tractatus Logico Philosophicus ([Wittgenstein61]) : "Ce dont on ne peut parler, vaut mieux le taire", mettant ainsi un terme à toute discussion supplémentaire qui serait par définition métaphysique, pour cause de non-sens, c'est-à-dire de 
non correspondance aux faits ou aux événements mondains. Cette ontologie particulière aura fait couler beaucoup de sueur sur le front des philosophes tout le long du vingtième siècle.

[2] Cela dit, je ne défends pas nécessairement une théorie épistémologique représentationaliste. Je ne pense pas, en effet, que le concept de connaissance soit réductible à celui de représentation mentale ou psychologique ou que le réel soit réductible à sa doublure (sur la question de la doublure, on consultera avec bénéfice les ouvrages de C. Rosset mentionnés à la bibliographie).

[3] Cela soulève d'innombrables questions classiques et moins classiques en épistémologie et en philosophie des sciences. En discuter n'est pas pertinent dans le contexte de cet article. Toutefois, je veux réaffirmer la posture réaliste de la science et de la connaissance en tant que postulat fondamental à toute activité scientifique et au contexte de découverte des connaissances fiables sur le monde. Le réalisme dont il est question ici est entendu dans son sens contemporain, pas en son sens médiéval. Ce dernier fera l'objet d'une discussion en temps opportun.

[4] QUINE, W.O., From a Logical Point of View [Quine53]. J'y reviens plus bas. Pour une discussion des implications logiques de ce critère de l'engagement ontologique, voir [Church58] pp. 1008-1014.

[5] Le recours au critère quinien de l'engagement ontologique des théories est fort répandu dans les ouvrages sur les ontologies SC et j'aurai l'occasion d'y revenir pour en fournir aussi une critique ; car ce recours me semble abusif.

[6] On trouve également dans Gruber [Gruber95] une définition canonique dont la portée, toutefois, est davantage méthodologique que théorique.

${ }^{\text {[7] }}$ Ce que confirment également [Smith04] et [Weltysmith01].

[8] Certains diront l'intension des concepts, ce qui est juste mais qui néanmoins pose de nouveaux problèmes théoriques de logique et de pragmatique des langages formels. En particulier celui-ci : si les termes du langage expriment les concepts du point de vue de leur intension, alors ces termes (ou catégories) sont analytiques car, suivant par exemple Carnap [Carnap56], ils expriment un contenu épistémique dans une langue L qui est un métalangage par rapport au langage descriptif (ou langageobjet) utilisé pour "remplir les classes". Mais ils sont également censés exprimer une classe de "choses", lesquelles sont pour ainsi dire subsumées sous le concept catégorique. Est-ce que seuls les termes catégoriques alors importent? Au surplus, quel est leur statut par rapport aux "choses" qu'ils regroupent ? On voit ainsi que non seulement les "ontologues" SC n'ont pas quitté le terrain médiéval de la querelle des universaux mais ils le reproduisent plutôt - tout en négligeant des questions de logique de leurs théories.

[9] Il va de soi que plusieurs concepts devraient être ici critiqués, tel celui de "chose" ("thing") servant à désigner de manière générique tout "existant" descriptible dans le langage $\boldsymbol{L}$ : la classe des "choses" est alors une classe universelle qui comprend elle-même comme élément puisque l'on est forcé de dire que "choses" existe en tant que classe et que donc elle fait partie de la classe des "existants" qu'elle contient.

[10] J'ignore si Sowa entend par "langage L" la même chose que Carnap. Dans ce qui suit, je ferai comme si ce n'était pas le cas qu'il utilise la même théorie du langage $L$ de Carnap. De toute manière, en bout de piste, ma critique est à mon avis valable dans l'un ou l'autre cas.

${ }^{[11]}$ Comme ce peut être également et éminemment le cas avec les mathématiques.

${ }^{[12]}$ Cette "existence de second niveau" est typique des "top-level ontologies". 
[13] Popper propose un schéma de base qui illustre sa thèse, et le fait correspondre, par analogie, à la thèse darwinienne de l'évolution des espèces : Soit un premier problème $P_{1}$, une théorie mise à l'essai $T T$ (pour "tentative theory", je respecte ici le choix du traducteur de [Popper91]), l'élimination des erreurs EE et un nouveau problème $P_{2}$; alors le schéma du développement des connaissances scientifiques est :

$P_{1} \rightarrow \mathrm{TT} \rightarrow \mathrm{EE} \rightarrow \mathrm{P}_{2}$. Avec ce schéma, seule la théorie la mieux adaptée survivra à la procédure de falsification. Elle ne sera pas vraie pour autant, conservant toujours le statut pérenne d'être falsifiable. Pour une critique du darwinisme appliquée à la théorie du progrès scientifique et de la philosophie, voir [Cohen86].

${ }^{[14]}$ En plus du titre déjà cité voir également à ce sujet : [Popper73], [Popper85].

[15] Popper écrit ([Popper91], pp. 248-249, italiques originaux) : "[...] l'apprentissage du langage joue un rôle essentiel chez l'être humain et il consiste, principalement, à apprendre à saisir des contenus de pensée objectifs (comme les appelait Frege)."

[16] " $\exists x: F x "$ n'entretient aucun engagement spécifique à propos de l'existence de Pégase. En tant qu'énoncé logique il est lié à l'ontologie de la logique. Par contre, s'il devait, par convention ou autrement, signifier une entité comme Pégase à l'intérieur d'une théorie quelconque, alors il jouerait le rôle d'un synonyme de Pégase et, donc, celui d'un nom. Cette distinction n'est pas comprise par Sowa.

${ }^{[17]}$ Ce concept mérite une attention spéciale. Une bonne définition, analytique et opératoire, se trouve dans [HoudéA198].

${ }^{[18]}$ Voici une liste de projets totalement arbitraire :

http ://www.m-w.com

http ://dictionary.reference.com

http ://www.foodsubs.com.

[19] Ce qui suppose au préalable d'avoir défini les concepts de données, information et connaissance ([Floridi99], p.106). Or, 1) l'auteur ne fait aucune différence entre les différentes formes de données (v. [Nadeau99]) ; 2) son concept d'information se résume à "donner forme à une donnée", ce qui le rapproche de la définition classique voire étymologique du terme ; 3) son concept de connaissance comme étant une information expliquée (sémantiquement augmentée par la compréhension humaine) mériterait d'être revu et corrigé à la lumière d'une phénoménologie post-husserlienne (v. [PetitotAl00]).

\section{Références}

\section{Références bibliographiques}

\section{[Andler92]}

Andler, D. (sous la direction de), Introduction aux sciences cognitives, Paris: Gallimard, Coll. Folio/Essai, 1992.

\section{[BarHillel64]}

Bar-Hillel, Y., Language and Information, Reading (Mass.) : Addison-Westley, 1964.

\section{[Bunge83]}

Bunge, M., Épistémologie, Paris : Maloine S.A. Éditeur, 1983. 


\section{[Bunge73]}

Bunge, M., Method, Model and Matter, Dordrecht : D. Reidel, 1973.

\section{[Carnap56]}

Carnap, R., Meaning and Necessity, Chicago : University of Chicago Press, 1956 (1947).

\section{[Carr77]}

Carr, B., "Popper's Third World”, The Philosophical Quarterly, Vol. 27, No. 108, (Jul. 1977), pp. $214-$ 226.

\section{[Church58]}

Church, A., "Ontological Commitment”, The Journal of Philosophy LV, 1958, pp. 1008-1014.

\section{[Church84]}

Church, R., "Popper's 'World 3'and the Problem of the Printed Line", Australasian Journal of Philosophy, Vol. 62, No. 4, Dec. 1984, pp. 378-391.

\section{[Cohen80]}

Cohen, L. J., "Some Comments on the Third World Epistemology", The British Journal of Philosophy of Science, 31, 1980, pp. 175-180.

\section{[Cohen86]}

Cohen, L. J., The Dialogue of Reason. An Analysis of Analytical Philosophy, Oxford: Clarendon Press, 1989 (1986).

\section{[Crosson67]}

Crosson, F. J., SAYRE, Kenneth M. (Eds), Philosophy and Cybernetics, Notre-Dame : University of Notre-Dame Press, 1967.

\section{[Davidson84]}

Davidson, D., Inquiries into Truth and Interpretation, Oxford : Clarendon Press, 1984.

\section{[Estivals02]}

Estivals, R., Théorie générale de la schématisation 1. Épistémologie des sciences cognitives, Paris : L'Harmattan, 2002.

\section{[Floridi04]}

Floridi, L. (Ed.), Philosophy of Computing and Information, Oxford (UK) : Blackwell Publishing, 2004.

\section{[Floridi99]}

Floridi, L., Philosophy and Computing. An introduction, London : Routledge, 1999.

\section{[Frege71]}

Frege, G., Écrits logiques et philosophiques, Paris : Seuil, 1971.

\section{[Gilroy85]}

Gilroy, J. D., “A Critique of Karl Popper's World 3 Theory “, The Modern Schoolman ; A Quarterly Journal of Philosophy, LXII, March 1985, pp. 185-200. 


\section{[George79]}

George, F. H., Philosophical Foundations of Cybernetics, Chatham : Abacus Press, 1979.

[Granger92]

Granger, G.-G., La vérification, Paris : Éditions Odile Jacob, 1992, coll. Philosophie.

\section{[Gruber95]}

Gruber, T. R., “Towards principles for design of ontologies used for knowledge sharing”, Int. J. HumanComputer Studies 43 (1995) pp. 907-928.

\section{[HoudéA198]}

Houdé, O. et al., Vocabulaire de sciences cognitives, Paris : PUF, coll. Psychologie et sciences de la pensée, 1998.

\section{[Keuth74]}

Keuth, H., "Objective Knowledge Out of Ignorance : Popper on Body, Mind and the Third World", Theory and Decision, 5, 1974, pp. 391-412.

\section{[Klemke79]}

Klemke, E.D., "Karl Popper, Objective Knowledge and the Third World”, Philosophia, Vol. 9, 1979, pp. 454-62.

\section{[Krausz74]}

Krausz, M., "Popper's Objective Knowledge”, Dialogue, vol. 13, no. 12, 1974, pp. 347-351.

\section{[Nadeau99]}

Nadeau, R., Vocabulaire technique et analytique de l'épistémologie, Paris: Presses Universitaires de France, 1999, Collection Premier Cycle.

\section{[Neale93]}

Neale, S., Descriptions, Cambridge : M.I.T. Press, 1993.

\section{[Newell90]}

Newell, A., Unified Theories of Cognition, Cambridge (Mass.) : Harvard University Press, 1990.

\section{[Petit97]}

Petit, J.-L. (éd.), Les neurosciences et la philosophie de l'action, Paris : J.Vrin, 1997, coll. Problèmes et controverses.

\section{[PetitotAl00]}

Petitot, J., Varela, F. J., Pachoud, B., Roy, J.-M., Naturaliser la phénoménologie. Essais sur la phénoménologie contemporaine et les sciences cognitives, Paris : CNRS Éditions, coll. Communication, 2002.

\section{[Pojman99]}

Pojman, L. P., The Theory of Knowledge. Classical and Contemporary Readings, New York : Wadsworth Publishing Company, 1999. 
Revue sticef.org

\section{[PolanyiProsch75]}

Polanyi, M., Prosch, H., Meaning, Chicago : University of Chicago Press, 1975.

\section{[Polanyi58]}

Polanyi, M., Personal Knowledge : Toward A Post-Critical Philosophy, Chicago : University of Chicago Press, 1958.

\section{[Popper73]}

Popper, K. R., La logique de la découverte scientifique, Paris : Payot, 1973.

\section{[Popper85]}

Popper, K. R., Conjectures et réfutations, Paris : Payot, 1985.

\section{[Popper91]}

Popper, K. R.., La connaissance objective, Paris : Flammarion, 1991, coll. Champs.

\section{[Quine53]}

Quine, W. V. Orman, From a Logical Point of View, Cambridge (Mass.) : Harvard University Press, 1953.

\section{[Quine60]}

Quine, W. V. Orman, Word and Object, Cambridge : M.I.T. Press, 1960, 294 p.

\section{[Rosset76]}

Rosset, C., Le réel et son double, Paris : Gallimard, 1984 (Édition revue et augmentée ; Paris : Gallimard, 1976).

\section{[Rosset77]}

Rosset, C., Le réel. Traité de l'idiotie, Paris : Éditions de Minuit, 1977, 155 p.

\section{[Rosset79]}

Rosset, C., L'objet singulier, Paris : Éditions de minuit, 1979, 113 p.

\section{[Smith04]}

Smith, B., "Ontology", in FLORIDI, Luciano (Ed.), Philosophy of Computing and Information, Oxford (UK) : Blackwell Publishing, 2004, pp. 155-166.

\section{[Tarski72]}

Tarski, A., Logique, Sémantique, Métamathématique. 1923-1944, Paris : Armand Colin, 1972 (1931).

\section{[WeltySmith01]}

Welty, C., Smith, B. (Eds.), Formal Ontology and Information Systems, New York : ACM Press, 2001.

\section{[Wittgenstein61]}

Wittgenstein, L., Tractatus logico-philosophicus, Paris : Gallimard, 1961, coll. Idées. 


\section{- A propos des auteurs}

Philosophe spécialiste de la logique et de l'épistémologie des sciences sociales et de la communication, Jean Robillard enseigne à la Télé-université (Université du Québec). Ses recherches portent sur la théorie de l'information et de la communication. Elles visent à évaluer l'influence de ces disciplines sur le développement, la méthodologie et l'épistémologie des sciences sociales.

Adresse : Unité d'enseignement et de recherche Sciences humaines, Lettres et Communication Télé-université (Teluq), Université du Québec 4750, avenue Henri-Julien, bureau 100, Montréal (Québec) H2T 3E4

Courriel : jean_robillard@teluq.uquebec.ca

\section{Référence de l'article :}

Jean ROBILLARD, Ph.D., Ontologies : antinomies, contradictions et autres difficultés épistémologiques du concept, Revue STICEF, Volume 11, 2004, ISSN:1764-7223, mis en ligne le 15/10/2004, http://sticef.org

(C) Revue Sciences et Technologies de 1'Information et de la Communication pour l'Éducation et la Formation, 2004 